\section{El papel de la universidad pública en la salud bucal de poblaciones excluidas}

\section{The public university role on excluded populations' oral health}

\section{Sr. Editor.}

Esta carta tiene como objetivo describir cómo una universidad pública, a través de sus actividades de extensión, puede actuar en comunidades que viven en exclusión social y mostrar las condiciones de salud bucal correlacionadas con el perfil socioeconómico de esas familias.

La actividad de extensión denominada "Acción Integradora en Salud" desarrolló acciones interdisciplinarias e intersectoriales dirigidas a la educación y capacitación de académicos y profesionales comprometidos con la realidad social y regional, de acuerdo con el perfil solicitado por las directrices curriculares nacionales y la política del Brasil Sin Miseria y del SUS (Sistema Único de Salud) en una población en las afueras del municipio de Viamão, Rio Grande do Sul, donde viven 1500 familias invasoras, cuya actividad es el reciclaje de basura como su principal fuente de ingresos (CENSO, 2010) ${ }^{1}$. Este programa de extensión expresa la postura de la universidad pública frente a la población excluida, y su función básica se caracteriza por ser la productora y socializadora del conocimiento, con el objetivo de intervenir en la realidad, permitiendo acuerdos y acciones colectivas entre esta y la población, enfocándose en los problemas sociales con el objetivo de encontrar soluciones a través de la investigación de campo desarrollada en educación y salud aplicada, con el objetivo de retroalimentar el proceso de enseñanza-aprendizaje en su conjunto, e intervenir en una realidad concreta.

En las últimas décadas, la condición social ha sido enfatizada como un determinante en la situación de la salud bucal. Los estudios han demostrado que la disminución de la caries dental ha sido acompañada por la polarización de la enfermedad en los grupos menos privilegiados. Varios estudios han abordado la asociación entre el desarrollo social y la caries dental, tanto en el campo individual como en el colectivo. Estos estudios revelan que la prevalencia de caries dental expresada por el índice CPOD es significativamente peor para las poblaciones con bajo nivel socioeconómico ${ }^{2}$. Los bajos ingresos pueden estar asociados con el nivel de educación, el valor atribuido a la salud, el estilo de vida y el acceso a la información sobre cuidados de la salud. Como consecuencia, el ingreso familiar puede ser un factor indirecto para la susceptibilidad a la caries ${ }^{3}$.

Esta actividad de extensión logró prestar atención a la educación sanitaria de 1501 personas de la Villa Augusta Meneguine, del municipio de Viamão, Rio Grande do Sul, Brasil, después de evaluar y discutir sus necesidades. El trabajo se realizó a través de visitas domiciliarias realizadas en

\section{Carta al Editor}

Márcia Cançado Figueiredo 1,a

${ }^{1}$ Universidade Federal do Rio Grande do Sul, Faculdade de Odontologia, Río Grande del Sur, Brasil.

${ }^{a}$ Doctora en Odontopediatría.

\section{Correspondencia:}

Márcia Cançado Figueiredo: mcf1958@gmail.com Rua Cananéia 255-310 Vila Jardim, Porto Alegre, Rio Grande do Sul, Brasil CEP 91.330.580

ORCID: 0000-0002-4279-5417

\section{Conflicto de intereses: ninguno}

Recibido: 31/03/20

Aprobado: 05/04/20

Publicado: 09/05/20

(c) Los autores. Este artículo es publicado por la revista Odontología Sanmarquina de la Facultad de Odontología, Universidad Nacional Mayor de San Marcos. Este es un artículo de acceso abierto, distribuido bajo los términos de la licencia Creative Commons Atribucion - No Comercia_Compartir Igual 4.0 Internacional. (http://creativecommons.org/licenses/by-nc-sa/4.0/) que permite el uso no comercial, distribución y reproducción en cualquier medio, siempre que la obra original sea debidamente citada. 
la referida Villa los días sábado, en las cuales los residentes de las casas visitadas respondieron a un cuestionario estandarizado, que contenía información socioeconómica del jefe de la familia, además de datos sobre la salud general, nutrición, peso y presión arterial de todos los miembros de la familia (Figura 1).

Las condiciones de salud bucal de estos residentes se evaluaron utilizando los criterios de presencia o ausencia de placa visible y sangrado gingival. También se registró el número de dientes cariados y perdidos, después del cepillado supervisado, enfatizando el proceso de enseñanza-aprendizaje. Estos exámenes orales fueron realizados por estudiantes de odontología utilizando luz artificial en los ambientes más claros de las casas (Figura 2).

Los datos recopilados de esta población se registraron en una hoja de cálculo Excel, se evaluaron y se trazó una estrategia de acción colectiva de salud de extensión universitaria que fuera desarrollada con esa comunidad. Esto se debió a que la población intervenida se caracterizó por vivir debajo de la línea de pobreza, porque las familias sobrevivían con menos de un salario mínimo por mes y la mayoría estaba compuesta de 3 a 5 residentes por casa. Esto refleja la realidad vivida también por 16,2 millones de personas que se encuentran en extrema pobreza, según los datos del último Censo brasileño del 2010, del Instituto Brasileño de Geografía y Estadística (IBGE) ${ }^{1}$. Uno de cada diez brasileños vive en condiciones de extrema pobreza, siendo el noreste $(18,1 \%)$ y norte $(16,8 \%)$ las regiones del país con el mayor número de personas en esta condición y las regiones del medio oeste $(4 \%)$, sudeste $(3,4 \%)$ y sur $(2,6 \%)$ con las de menor número ${ }^{1}$.
Esta realidad encontrada en la comunidad de la Villa, reflejaba una relación inversamente proporcional entre el ingreso de sus residentes y su frecuencia de consumo de azúcar entre comidas. Curiosamente, Levy-Costa et al. ${ }^{4}$ también encontraron que el consumo de azúcar en la clase de ingresos más bajos fue $50 \%$ más alto que el observado en la clase de ingresos más altos, y Tomita et al. ${ }^{5}$ apoyaron la hipótesis de que el nivel socioeconómico influye en la preferencia por el azúcar y esto, a su vez se asoció con la prevalencia de caries dental en la dentición decidua.

La presencia de caries dental fue significativa en casi todas las personas de la Villa. Sin embargo, aún más preocupante fueron los datos sobre la pérdida de dientes, que se encontraban en el segmento de los adultos jóvenes que ciertamente tendrían dificultades para masticar, hablar, cambios en el comportamiento, insatisfacción con la apariencia, baja aceptación social y dificultad para acceder al mercado de trabajo, causando un fuerte impacto en la calidad de vida (Vargas y Paixáo) ${ }^{6}$.

De hecho, fue posible percibir la necesidad de acciones interdiscilinarias de extensión en salud para promover la salud a través de la atención a una población desatendida y estas acciones deberían ser adoptadas por las universidades públicas para que haya una contribución a la sociedad y al mismo tiempo que preparan a sus estudiantes de pregrado para una formación profesional insertada en la realidad de cada país.

Finalmente, se puede concluir que la universidad pública tiene un papel fundamental no solo en la educación del estudiante, sino también, con sus actividades

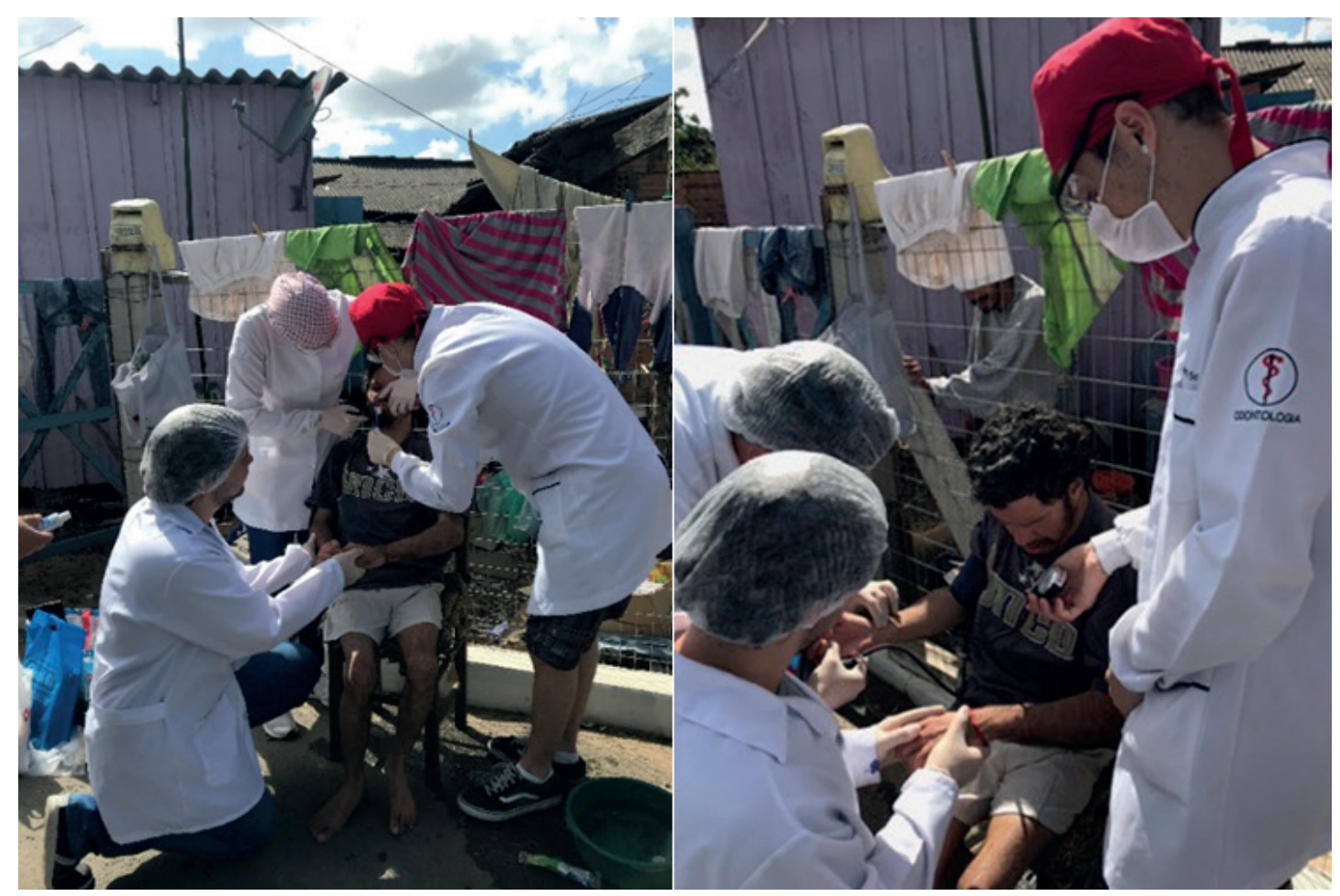

Figura 1. Exámenes realizados durante una visita domiciliaria 


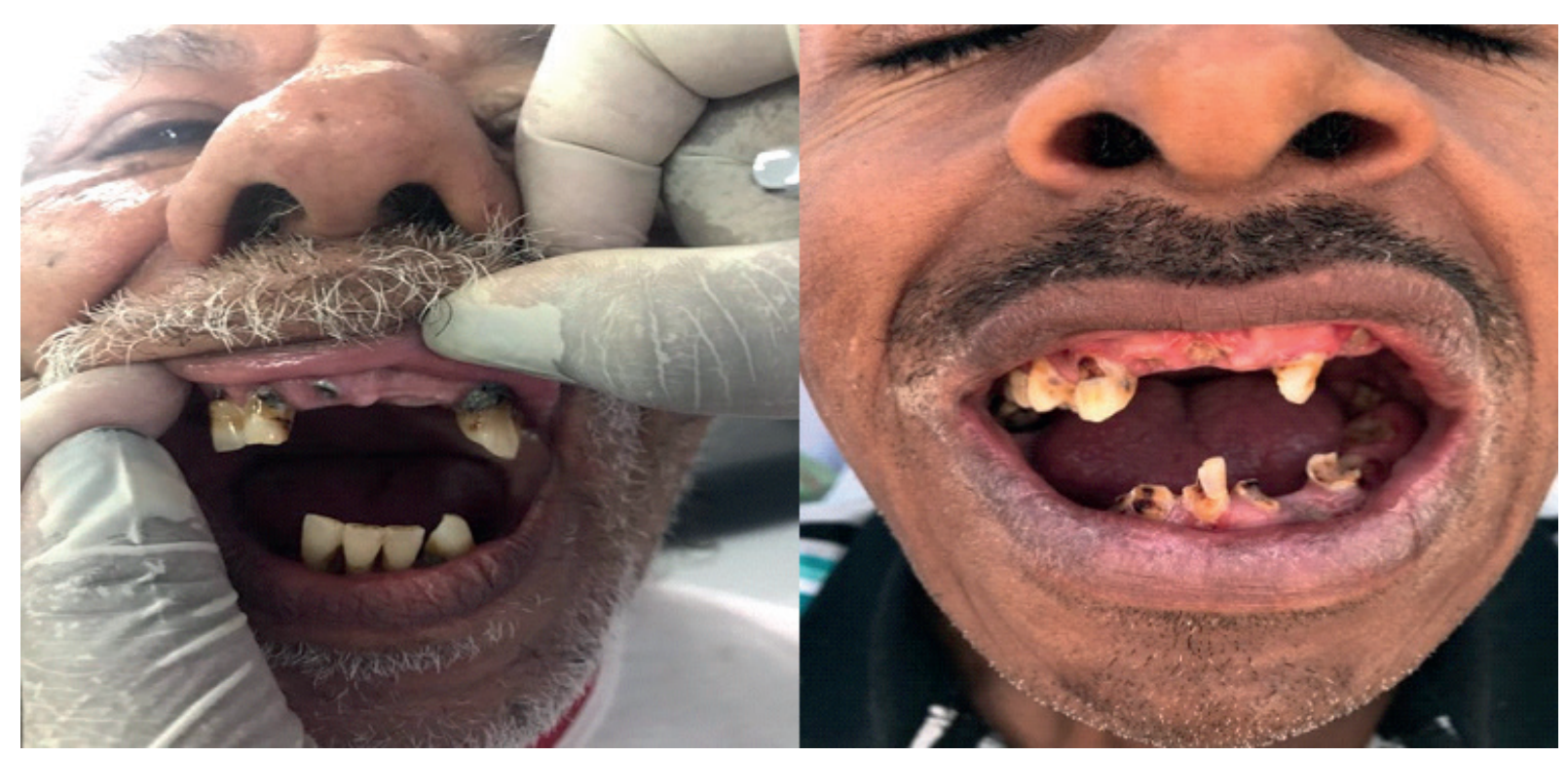

Figura 2. Ejemplos de las condiciones de salud bucal de los pobladores de la Villa, evaluados durante la actividad de extensión

de extensión, en la identificación de las necesidades de salud de las comunidades y, por lo tanto, para ayudarlos en lo que más necesitan.

\section{Referencias bibliográficas}

1. Baldani MH, Vasconcelos GA, Antunes Ferreira LJ. Associação do índice CPO-D com indicadores socioeconômicos e de provisão de serviços odontológicos no Estado do Paraná, Brasil. Cad Saúde Pública 2004;20(1):143-52.

2. Lucas SD, Portela MC, Mendonça LL. Variaçôes no nível de cárie dentária entre crianças de 5 e 12 anos em Minas Gerais, Brasil Cad Saúde Pública 2005;21(1):55-63.

3. Instituto Brasileiro de Geografia e Estatística (IBGE). Indicadores sociais municipais: uma análise dos resultados do universo do censo demográfico, análise preliminar da distribuiçấo e diferenciais de rendimento. Rio de Janeiro. 2011. [Consultado el 30 de marzo del 2020]. Accesible en: https://biblioteca.ibge.gov.br/visualizacao/livros/ liv54598.pdf
4. Levy-costa RB, Sichieri R, Pontes NS, Monteiro CA. Disponibilidade domiciliar de alimentos no Brasil: distribuição e evolução (1974-2003). Rev Saúde Publica 2005;3(4):530-40.

5. Tomita NE, Nadanovsky P, Vieira LA, Lopes ES. Preferências por alimentos doces e cárie dentária em pré-escolares. Rev Saúde Pública 1999;3(6):542-6.

6. Vargas DM, Paixão HH. Perda dentária e seu significado na qualidade de vida de adultos usuários de serviço público de saúde bucal do Centro de Saúde Boa Vista, em Belo Horizonte. Ciênc saúde coletiva 2005;10(4):1015-24. 
